\title{
miRNA-504 inhibits p53-dependent vascular smooth muscle cell apoptosis and may prevent aneurysm formation
}

\author{
XUE CAO, ZHENGUO CAI, JUNYAN LIU, YANRU ZHAO, XIN WANG, XUEQI LI and HONGYUAN XIA \\ Department of Cardiology, The Fourth Affiliated Hospital of Harbin Medical University, \\ Harbin, Heilongjiang 150006, P.R. China
}

Received April 7, 2016; Accepted March 23, 2017

DOI: $10.3892 / \mathrm{mmr} .2017 .6873$

\begin{abstract}
Abdominal aortic aneurysm (AAA) is a common disease that is associated with the proliferation and apoptosis of vascular smooth muscle cells (VSMCs). VSMCs are regulated by microRNAs (miRNA). The aim of the present study was to identify miRNA sequences that regulate aortic SMCs during AAA. miRNA-504 was identified using a miRNA PCR array and by reverse transcription-quantitative polymerase chain reaction analysis, and its expression levels were observed to be downregulated in the aortic cells derived from patients with AAA when compared with controls. Transfection of SMCs with pMSCV-miRNA-504 vector was performed, and cell proliferation and the expression levels of proliferating cell nuclear antigen (PCNA), replication factor C subunit 4 (RFC4), B-cell lymphoma-2 (Bcl-2) and caspase-3/9 were measured by western blotting. The mechanisms underlying the effects of miRNA-504 was then analyzed. The results demonstrated that overexpression of miRNA-504 significantly upregulated the expression levels of PCNA, RFC4 and Bcl-2, while caspase-3/9 expression was significantly inhibited when compared with non-targeting controls. In addition, miRNA-504 overexpression was observed to promote the proliferation of SMCs. The expression level of the tumor suppressor, p53, which is known to be a direct target of miRNA-504, was inhibited following transfection of SMCs with pMSCV-miRNA-504. In addition, the expression of the downstream targets of p53, p21 and Bcl-like protein-4, were significantly reduced following overexpression of miRNA-504. These results revealed the anti-apoptotic role of miRNA-504 in SMCs derived from patients with AAA via direct targeting of p53.
\end{abstract}

Correspondence to: Dr Hongyuan Xia, Department of Cardiology, The Fourth Affiliated Hospital of Harbin Medical University, 37 Yiyuan Road, Nangang, Harbin, Heilongjiang 150006, P.R. China E-mail: hyxia89@yeah.net

Key words: aneurysm, vascular smooth muscle cells, apoptosis, microRNA-504

\section{Introduction}

Abdominal aortic aneurysm (AAA) is a common disease that is correlated with dilatation of the aorta to $\geq 5 \mathrm{~mm}$ in diameter, and occurs below the renal arteries (1). Vascular degradation is closely associated with advanced age, being female, smoking and a family history of the condition (2). AAA is responsible for $\sim 15,000$ mortalities in the USA per annum, despite the recent reduction in prevalence and mortality due to decreased smoking rates, increased early detection and improved surgical procedures $(3,4)$. The incidence of AAA is 6 -fold higher in males $>60$ years of age and females $>65$ years of age. AAA develops due to extensive vascular inflammation combined with maladaptive remodeling of the aortic wall $(5,6)$. Although various clinical and laboratory studies have provided insights into the pathogenesis of AAA, the underlying mechanisms remain to be elucidated (7).

The pathogenesis of AAA is very complex; however, increasing evidence suggests that vascular smooth muscle cells (VSMC) are associated with the development of AAA. VSMCs provide a source of elastin, which serves an important role in maintaining the elasticity of the aortic wall $(8,9)$. However, during the development of AAA, proteolytic processes induce the degradation of the media layer, which leads to a reduction of elastin content and expansion of the aortic wall. The deficiency in elastin content subsequently leads to a compensatory increase in collagen synthesis, which contributes to remodeling of the AAA wall (10). This decrease in elastin has been attributed to the induction of senescence and apoptosis of VSMCs (11). AAA is characterized by degradation of the extracellular matrix, a potent inflammatory response, and increased oxidative stress in the abdominal aortic wall $(12,13)$. In addition, infiltration by inflammatory cells may accelerate apoptosis of VSMCs. Inhibition of apoptosis and SMC regeneration are considered to be desirable endpoints for the treatment of AAA. Through the identification of a large number of miRNA sequences with differences in expression levels between AAA samples and those derived from abdominal aortic tissues from age and sex-matched controls, a number of previous reports have indicated that microRNAs (miRNAs) may be involved in AAA (14-17).

miRNAs are a class of small non-coding RNAs that bind preferentially to the 3'-untranslated region (3'-UTR) of target mRNA sequences and transport them to the RNA-induced 
silencing complex, which results in the downregulation of gene expression by mRNA degradation and inhibition of translation (18). This process contributes to the regulation of crucial biological activities, including cell proliferation, apoptosis and differentiation (19,20). Despite the identification of $<1,000$ human miRNAs (21), it is now evident that $>30 \%$ of the genome is regulated by miRNAs (22). Previous studies have confirmed that miRNAs serve crucial roles in tumorigenesis, as well as the invasion and apoptosis of cancer cells in various human malignancies $(23,24)$. In addition, miRNAs have been demonstrated to target tumor suppressor genes inhibiting smooth muscle cell proliferation and migration, thus influencing angiogenesis $(25,26)$.

Although a considerable number of studies investigating miRNAs have been conducted, a limited number of miRNA expression profiling studies involving VSMCs from patients with AAA have been performed to date. Therefore, the present study investigated the expression patterns of miRNAs in aortic SMCs from patients with AAA, in order to identify potential miRNAs that are associated with the disease. A microarray-based genome-wide screening study was first performed, followed by analysis of the expression of individual miRNAs by reverse transcription-quantitative polymerase chain reaction (RT-qPCR). Bioinformatic analyses were conducted to predict gene targets of the identified miRNAs and determine their putative roles in AAA.

\section{Materials and methods}

Human aortic SMC samples. Aortic SMC specimens were obtained from patients undergoing AAA repair operations $(n=60)$ at the Department of Cardiology, The Fourth Affiliated Hospital of Harbin Medical University (Harbin, China) from January 2013 to December 2014. Aortic wall tissues and matched non-aneurysmal aortic samples were snap-frozen in liquid nitrogen and stored at $-80^{\circ} \mathrm{C}$ until RNA was extracted. Written informed consent was obtained from all study percipients. The present study received ethical approval from the Independent Ethics Committee of Shanghai First People's Hospital (Shanghai, China), and all experiments were conducted in accordance with the Declaration of Helsinki.

Cell culture and RNA isolation. SMCs were propagated in SmGM-2 Smooth Muscle Growth Medium-2 (Lonza Group, Basel, Switzerland) containing $10 \%$ fetal bovine serum (FBS) according to the manufacturer's instructions. Cells were maintained in a humidified atmosphere at $37^{\circ} \mathrm{C}$ and $5 \%$ $\mathrm{CO}_{2}$. After $48 \mathrm{~h}$, cells were harvested and total RNA isolated using the mirVana ${ }^{\mathrm{TM}}$ miRNA Isolation kit (Ambion; Thermo Fisher Scientific, Inc., Waltham, MA, USA) according to the manufacturer's instructions.

Preparation of miRNA and total RNA and RT-qPCR analysis. The quality of RNA and miRNA in samples was assessed using a NanoDrop spectrophotometer (NanoDrop; Thermo Fisher Scientific, Pittsburgh, PA, USA), and verified using the Agilent 2100 Bioanalyzer (Agilent Technologies, Inc., Santa Clara, CA, USA; all sample RNA integrity values were >9). For quantification of gene transcription, $10 \mu \mathrm{l}$ of cDNA containing $10^{10}$ copies of RNA was generated using the Moloney murine leukemia virus reverse transcriptase (primers were sense: 5'-NNG/T; anti-sense: 5'-A/CNN), and then amplified on the ABI PRISM 7900HT Sequence Detection System (Applied Biosystems; Thermo Fisher Scientific, Inc.) using TaqMan primers (TM001973; forward ACCCTGGTCTGCACTCTA TCT and reverse TGCCCTCTGTATGGGAAACC; Applied Biosystems; Thermo Fisher Scientific, Inc.). The expression of miRNA sequences was quantified using TaqMan. miRNA Reverse Transcription kits (SM-10153) and TaqMan miRNA assays (Applied Biosystems; Thermo Fisher Scientific, Inc.). Thermal cycling parameters were $94^{\circ} \mathrm{C}$ for $30 \mathrm{sec}, 57^{\circ} \mathrm{C}$ for $30 \mathrm{sec}$ and $72^{\circ} \mathrm{C}$ for $30 \mathrm{sec}$. For miRNAs quantification, $10 \mathrm{fmol}$ was diluted to $0.01 \mathrm{amol}$ and spiked into a constant amount of carrier RNA, and the Cq values were measured.

miRNA array hybridization. RNA was purified and pooled from samples of treatments using TRIzol reagent (Invitrogen; Thermo Fisher Scientific, Inc.) according to the manufacturer's protocol. This method yielded an average of $30 \mu \mathrm{g}$ total RNA from $10^{6}$ cells. Hybridized microarray was performed by the Agilent Human miRNA 8x15k Array 2.0 (cat. no. G4470B; Agilent Technologies, Inc.) according to manufacturer's protocol, and the quantity of RNA which was used array hybridization was $5 \mu \mathrm{g}$. The microarray contained 830 miRNA probes from the Sanger database (version 10.1, http://www.sanger.ac.uk/). Arrays were scanned using the Agilent Microarray Scanner System and Feature Extractor Software (version 9.5.1; Agilent Technologies, Inc.).

miRNA array analysis. Quality control (QC) reports were generated using the Agilent Feature Extraction software, and the array data were analyzed using GeneSpring GX software (version 10.0.2; Agilent Technologies, Inc.). Arrays were examined by QC metrics and principal components analysis, as well as hierarchical agglomerative clustering. Two chips clustered separately and were therefore excluded. The remaining arrays were subject to one-way analysis of variance (threshold, $\mathrm{P}<0.05$ ), with a Benjamini-Hochberg post hoc correction for multiple testing. Significant miRNAs were required to exhibit a $>2$-fold increase or decrease in expression compared with the control at the $72 \mathrm{~h}$-time point. Pairwise analysis was performed between the control and the 72 h-time point using statistical analysis of microarrays with false detection ratio of $<1$. TargetScan (version 5.1, http://www .targetscan.org/worm_12/docs/help.html.) and PicTar version 4.0.24 (http://www.pictar.org/.) software programs were used to identify putative miRNA gene targets (27-29).

Validation of miRNA array results. In order to validate the expression levels of miRNA sequences identified by microarray analysis, further RT-qPCR was performed. Briefly, samples were incubated for $60 \mathrm{~min}$ at $42^{\circ} \mathrm{C}$, followed by heat inactivation of the reverse transcriptase for $5 \mathrm{~min}$ at $95^{\circ} \mathrm{C}$ prior to storage at $4^{\circ} \mathrm{C}$. To convert mRNA to cDNA, total RNA was reverse transcribed using the High-Capacity cDNA reverse transcription kit (cat. no. 4368813; Applied Biosystems; Thermo Fisher Scientific, Inc.). Reaction mixtures were incubated at $25^{\circ} \mathrm{C}$ for $5 \mathrm{~min}$, followed by $37^{\circ} \mathrm{C}$ for $120 \mathrm{~min}$ then $85^{\circ} \mathrm{C}$ for $5 \mathrm{~min}$, prior to storage at $-20^{\circ} \mathrm{C}$. Standard thermocycling conditions were used and reactions were performed 
in triplicate with the corresponding positive and negative controls. Primer sequences for Let-7c were GCTGACTGA AGATATGATAAGG and ATGACACATTACCTTCTTGC; for miR-449a CACGAGAATCGGCAGTGAC and AACACT GTCTACTCCGAAAGACTCC; for miR-144-3p GCTGGG ATATCATCATATACTG and CGGACTAGTACATCATCT ATACTG; for miR-192-5p CTGACCTATGAATTGACAGCC and TGACCTATGGAATTGGCAG; for miR-504 TCTACA CAGGTTGGGATCGG and CGGGACAAGTGCAATACC ATA; for miR-542-3p GAGCTAGCACTTCCCGAGC and TAGCTGTCTGCCCCTTGTCT, and the primer for endogenous control was TTGCGGGTCTAATCACCGATT and TCG GTGATTAGACCCGCAATT. The thermal cycling parameters were as follows: $95^{\circ} \mathrm{C}$ for $5 \mathrm{~min}, 45$ cycles of $10 \mathrm{sec}$ at $95^{\circ} \mathrm{C}, 20 \mathrm{sec}$ at $60^{\circ} \mathrm{C}$ and $15 \mathrm{sec}$ at $72^{\circ} \mathrm{C}$; followed by a melting curve of $10 \mathrm{sec}$ at $95^{\circ} \mathrm{C}, 60 \mathrm{sec}$ at $60^{\circ} \mathrm{C}$ and continued melting. A SYBR-Green Master mix (Thermo Fisher Scientific, Inc.) was used. Target gene expression was quantified using the $2^{-\Delta \Delta \mathrm{Cq}}$ method (30). All RT-qPCR analyses for the quantification of miRNA expression levels were completed within $24 \mathrm{~h}$ of the reverse transcription step.

miRNA transfection. The DNA fragments encoding miRNA-504 or control miRNA sequences and the miRNA-504 inhibitor or control small interfering-RNA sequences (NC) were purchased from GeneCopoeia, Inc. (Rockville, MD, USA), and were inserted into the pMSCV vector (Clontech Laboratories Inc., Mountain View, CA, USA). Transfection was performed with Lipofectamine 2000 transfection reagent (Invitrogen; Thermo Fisher Scientific, Inc.) according to manufacturer protocols (PanVera; Thermo Fisher Scientific, Inc.). The number of cells transfected was $1.5 \times 10^{4} \mathrm{~cm}^{-2}$, and the quantity of plasmid DNA was $1.5 \mathrm{mg}$ in a volume of $10 \mu 1$. Aortic SMCs were cultured in medium containing $10 \%$ FBS for $24 \mathrm{~h}$ prior to transfection with miRNA sequences. The viral vectors were added to the medium for transfection. At $48 \mathrm{~h}$ following transfection, cells were cultured in medium containing $1 \mu \mathrm{g} / \mathrm{ml}$ puromycin to select for positive transfection clones. In order to maintain stable expression of the inserted sequence, the positive cells were cultured in medium containing $0.5 \mu \mathrm{g} / \mathrm{ml}$ puromycin. The primer sequence for miRNA-504 was 5'-GGT GAAGGTCGGTGTGAACG-3' (forward), and 5'-TGGAGG CCATGTAGGCCATG-3' (reverse).

Luciferase reporter assay. In order to determine the effect of miRNA-504 on the 3'-UTR of the p53 mRNA sequence, the Dual-Luciferase Reporter 1000 assay system (Promega Corporation, Madison, WI, USA) was utilized. Briefly, 1,000 to 15,000 cells were cultured in 24-well cell culture plate until the cells reached $70 \%$ confluence. Cells were co-transfected using a co-transfected reagent from Qiagen Sciences, Inc. (Gaithersburg, MD, USA) with hsa-miRNA-504 or control miRNA sequences and the 3'-UTR of p53 (mutant of p53 3'-UTR was 5'-ACUUGUUUUAUAGCAUCUACU-3'). The quantities of hsa-miR-504 and control miRNA were calculated using $2^{-\Delta \Delta \mathrm{Cq}}$ method (30). At $48 \mathrm{~h}$ following transfection, cells were harvested and firefly and Renilla luciferase activities were assayed. Lipofectamine 2000 (Invitrogen; Thermo Fisher Scientific, Inc.) and a Dual-Luciferase reporter assay system (Promega Corporation) were used for sequential measurement of firefly and Renilla luciferase activities. Quantification of luciferase activities and calculation of relative ratios were carried out using a luminometer (TD-20/20; Turner Designs, Sunnyvale, CA, USA); at least three independent transfections were performed in triplicate. The results were normalized to Renilla luciferase activity.

Western blot analysis. After $24 \mathrm{~h}$, whole aortic cell lysates were extracted using radioimmunoprecipitation assay lysis buffer (150 mM Tris-HCl, $50 \mathrm{mM} \mathrm{NaCl}, 1 \%$ Nonidet P-40 and $0.1 \%$ Tween-20) and the protein concentration was determined using the Bradford assay (Bio-Rad Laboratories, Inc., Hercules, CA, USA). Protein extracts ( $30 \mu \mathrm{g})$ were resolved by SDS-PAGE (6 or $7.5 \% \mathrm{w} / \mathrm{v})$ and transferred electrophoretically to polyvinylidene difluoride membranes. Following blocking of membranes with dried non-fat milk $(5 \% \mathrm{w} / \mathrm{v} ; 1 \mathrm{~h}$ at room temperature), membranes were probed with the following primary antibodies overnight at $4^{\circ} \mathrm{C}$ : Anti-proliferating cell nuclear antigen (anti-PCNA; cat. no. sc-25280; 1:200, Santa Cruz Biotechnology, Inc., Dallas, TX, USA), anti-replication factor C subunit 4 (RFC4) (cat. no. sc-28301; 1:1,000, Santa Cruz Biotechnology, Inc.), anti-caspase-3 (cat. no. sc-7272; 1:500; Santa Cruz Biotechnology, Inc.), anti-caspase-9 (cat. no. sc-81589; 1:500; Santa Cruz Biotechnology, Inc.), anti-B-cell lymphoma-2 (Bcl-2) (cat. no. sc-23960; 1:1,000; Santa Cruz Biotechnology, Inc.), the anti-p53 rabbit monoclonal antibody (53 kD cat. no. 8712; 1:1,000; Cell Signaling Technology, Inc., Danvers, MA, USA), the anti-p21 rabbit monoclonal antibody (21 kD; cat. no. 2947; 1:1,000; Cell Signaling Technology, Inc.) and anti-bcl-2-like protein 4 (Bax; cat. no. 2772; 1:1,000; Cell Signaling Technology, Inc). Membranes were subsequently incubated with the peroxidase-conjugated goat anti-rabbit IgG secondary antibody (cat. no. A0208; Beyotime Institute of Biotechnology, Shanghai, China) at 1:2,000 for $1 \mathrm{~h}$ at room temperature. Immunoreactive bands were normalized to $\beta$-actin and visualized using an ECL kit (Amersham Pharmacia Biotech Inc., Piscataway, NJ USA) then quantified using ImageQuant software version 5.2 (GE Healthcare Life Sciences, Chalfont, UK).

Determination of cell proliferation. Treated and untreated aortic cells were seeded at $3.7 \times 10^{4}$ cells $/ \mathrm{cm}^{3}$ in 96 -well plates and incubated for 24, 48, 72, 96 and $120 \mathrm{~h}$. A CCK-8 assay (Dojindo Molecular Technologies, Inc., Kumamoto, Japan) was performed by adding $10 \%$ CCK- 8 solution (v/v) to each well and incubating cells for $1 \mathrm{~h}$ at $37^{\circ} \mathrm{C}$. The optical density values for each well were measured at a wavelength of $450 \mathrm{~nm}$ using a microplate reader (Bio-Rad Laboratories, Inc.). All experiments were performed in triplicate and repeated at least three times.

Cell apoptosis assay. Apoptosis was analyzed using the Annexin V-fluorescein isothiocyanate (FITC; $5 \mu 1$ )/propidium iodide (PI; $5 \mu \mathrm{l}$; both from Invitrogen; Thermo Fisher Scientific, Inc.) staining method. The reagents were mixed with $500 \mu \mathrm{l}$ buffer and flow cytometry analysis was preformed according to manufacturer's instructions. Cells ( $2-4 \times 10^{5}$ cells/well) were cultured on $60-\mathrm{mm}$ diameter dishes and infected with the aforementioned viral vectors. At $48 \mathrm{~h}$ following transfection, cells were harvested by trypsinization, 
A

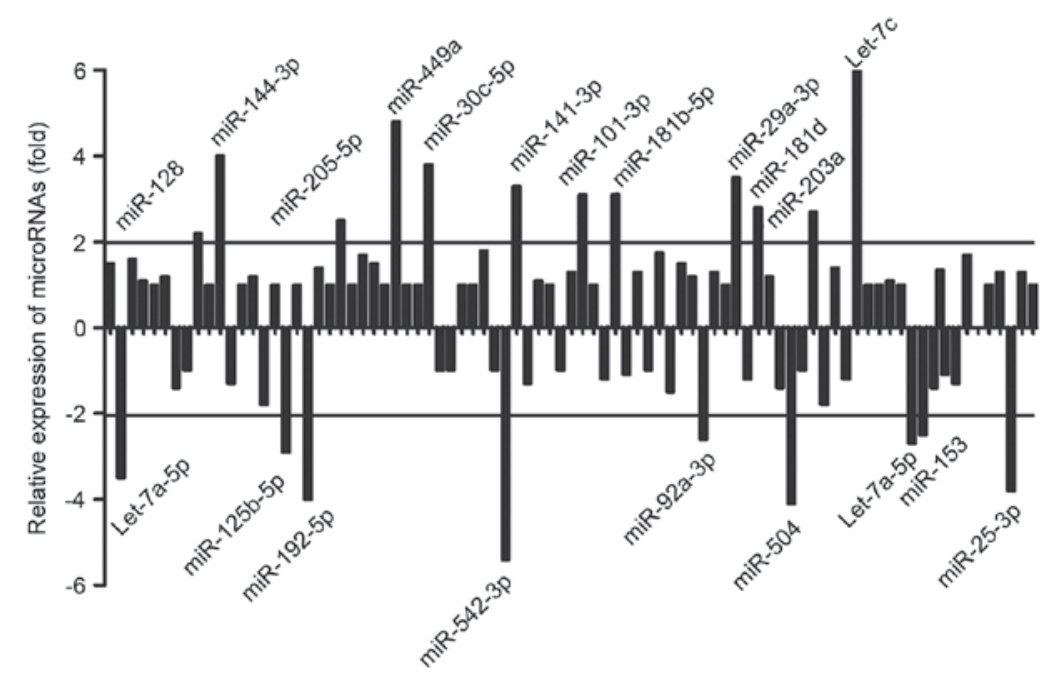

B

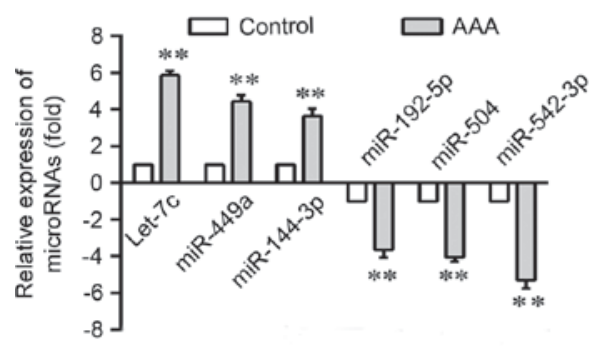

C

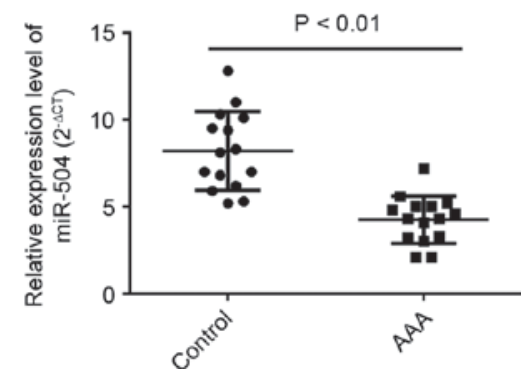

Figure 1. Identification and verification of the differential expression patterns of miRNAs in the aortic SMCs of patients with AAA. (A) Screening of miRNA sequences in serum samples from patients with AAA and paired controls using a PCR microarray. (B) Validation of microarray results for selected miRNAs that were significantly upregulated and downregulated in AAA samples when compared with controls, as determined by RT-qPCR analysis. To verify the accuracy of the microarray-based miRNA quantification, the six above-mentioned miRNAs (Let-7c, miR-499a, miR-144-3p, miR-192-5p, miR-504 and miR-542-3p) were re-examined using RT-qPCR. The results confirmed that the microarray data was reliable. (C) Confirmation of miRNA-504 expression in aortic SMCs derived from patients with AAA and paired controls by RT-qPCR analysis. ${ }^{* *} \mathrm{P}<0.01$ vs. controls. miRNA, microRNA; AAA, abdominal aortic aneurysm; RT-qPCR, reverse transcription-quantitative polymerase chain reaction.

washed with ice-cold phosphate-buffered saline (PBS), and fixed with ice-cold $70 \%$ ethanol for $>2 \mathrm{~h}$ at $-20^{\circ} \mathrm{C}$. The fixed cells were then washed with PBS and incubated with ribonuclease A $(20 \mu \mathrm{g} / \mathrm{ml}$ at final concentration; Sigma-Aldrich; Merck KGaA, Darmstadt, Germany) and PI (0.05 mg/ml; Sigma-Aldrich; Merck KGaA) at room temperature for $30 \mathrm{~min}$. Cells were then labeled with Annexin V-FITC (final concentration $0.25 \mu \mathrm{g} / \mathrm{ml}$ ) and PI (final concentration $12.5 \mu \mathrm{g} / \mathrm{ml}$ ) in the dark for $15 \mathrm{~min}$ at room temperature and analyzed by flow cytometry (BD Biosciences, San Jose, CA, USA). At least $2 \times 10^{4}$ cells were analyzed for each sample and the distribution of cells was analyzed using CellQuest ${ }^{\mathrm{TM}}$ software (version 3.3; BD Biosciences). The experiments were performed in triplicate.

Statistical analysis. Data are expressed as the mean \pm standard error of the mean from at least three repeated experiments. Differences between groups were analyzed using one-way analysis of variance followed by Tukey's test. All statistical analyses were conducted using SPPS software (version 20.0; IBM SPSS, Armonk, NY, USA). P<0.05 was considered to indicate a statistically significant difference.

\section{Results}

miRNA expression profiling of clinical samples. In order to identify miRNA sequences associated with AAA, the expression levels of 84 miRNAs that have been previously implicated in angiocardiopathy and cancer in AAA were investigated. Following analysis of the miRNAs that demonstrated differential expression patterns in AAA samples compared with control samples, a number of miRNAs that were reproducibly up- or downregulated in the aortic cells were identified. The criteria for differentially expressed miRNAs was based on the following two conditions: miRNA sequences should demonstrate a $>2$-fold alteration in gene expression when compared with the controls, and exclusion of miRNAs with low expression levels (quantification cycle, $>30$ ), as quantification by RT-qPCR analysis may produce unreliable results. Accordingly, 21 differentially expressed miRNAs were identified in aortic cells from patients with AAA (Fig. 1A). A total of 12 miRNAs were upregulated, while 9 were downregulated in aortic cells. Among these, the expression of 6 miRNAs, which were significantly altered when compared with the remaining miRNAs, was verified by RT-qPCR analysis (Fig. 1B). As 

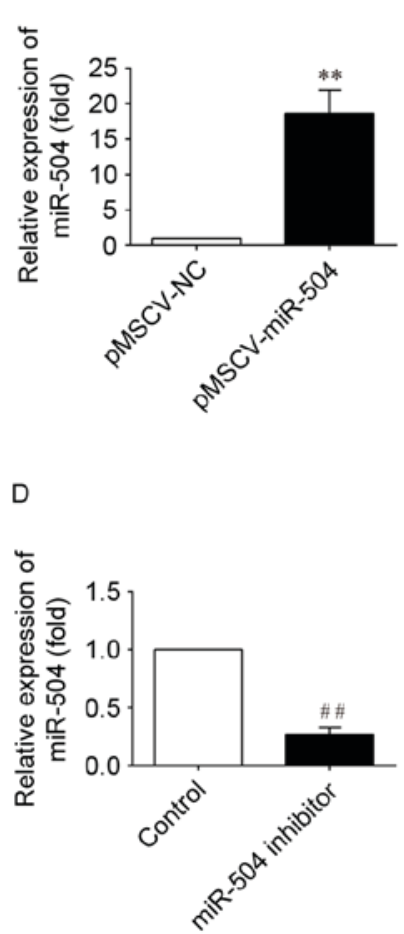

B

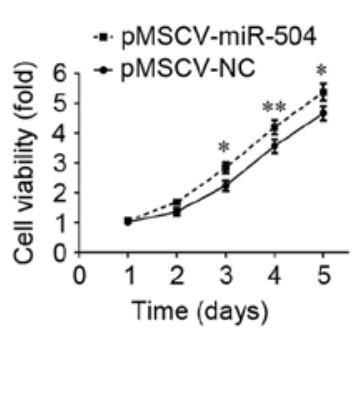

$\mathrm{E}$

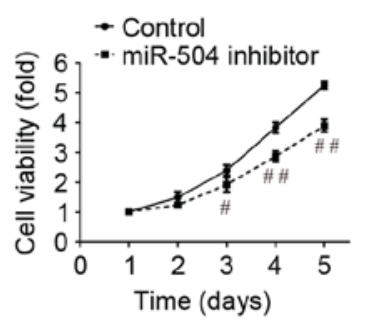

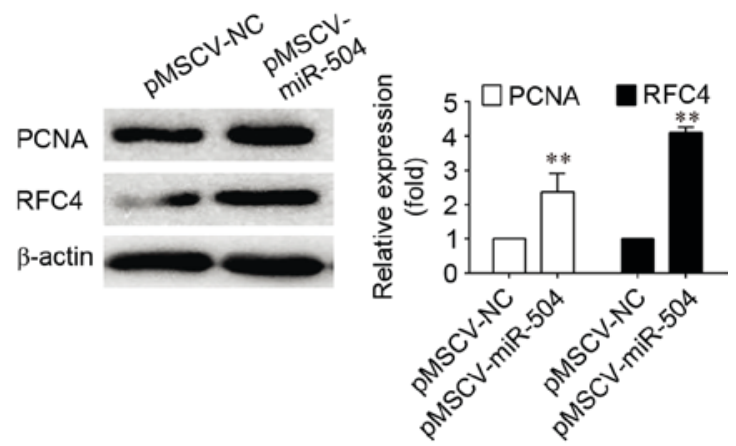

$\mathrm{F}$

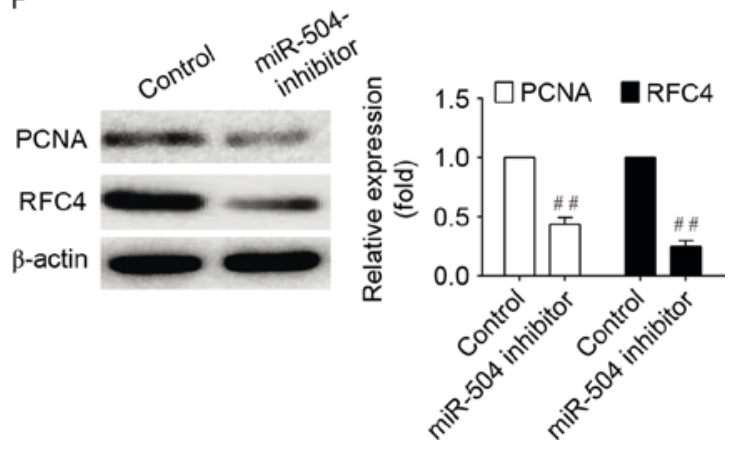

Figure 2. Effect of miRNA-504 on the proliferation of vascular SMCs. (A) The expression of miRNA-504, (B) the viability of aortic SMCs and (C) the protein expression levels of PCNA and RFC4 following transfection of aortic SMCs with pMSCV-miRNA-504 or pMSCV-NC vectors. (D) The expression of miRNA-504, (E) the viability of aortic SMCs and (F) the protein expression levels of PCNA and RFC4 following transfection of aortic SMCs with the miRNA-504 inhibitor or control. ${ }^{*} \mathrm{P}<0.05$ and ${ }^{* * *} \mathrm{P}<0.01$ vs. pMSCV-NC; ${ }^{*} \mathrm{P}<0.05$ and ${ }^{\# \#} \mathrm{P}<0.01$ vs. the control. miRNA, microRNA; SMCs, smooth muscle cells; NC, negative control; PCNA, proliferating cell nuclear antigen; RFC4, replication factor C subunit 4.

shown in Fig. 1C, the expression level of miRNA-504 was significantly higher in the AAA aortic SMCs when compared with matched normal controls $(\mathrm{P}<0.01)$. Therefore, the results suggest that miRNA-504 expression was significantly increased in AAA. In addition, previous studies have reported that miRNA-504 exhibits anti-apoptotic functions, and AAA is closely associated with apoptosis of smooth muscle cells $(31,32)$. The result indicated that miRNA-504 may be closely related with AAA and therefore the functional role of miRNA-504 was investigated further in the present study.

Effect of miRNA-504 on the proliferation of aortic SMCs. To examine the effect of miRNA-504 on the proliferation of SMCs, the proliferation of aortic cells infected with pMSCV vectors containing non-targeting control, miRNA-504 or miRNA-504 inhibitor sequences was examined using the CCK-8 assay. In the pMSCV-miRNA-504-transfected aortic cells, cell growth was significantly increased within 5 days when compared with negative controls $(\mathrm{P}<0.05$; Fig. 2). By contrast, transfection of cells with the miRNA-504 inhibitor, significantly inhibited cell proliferation when compared with the non-targeting controls $(\mathrm{P}<0.05$; Fig. 2). These results indicate that miRNA-504 may promote the proliferation of SMC cells.

In order to investigate the signaling pathways associated with the miRNA-504-mediated increase in SMC proliferation further, the expression levels of two genes involved in DNA replication, PCNA and RFC4 $(33,34)$, which serve as checkpoints of DNA replication, were examined. The western blotting results demonstrated that the PCMA and RFC4 expression was significantly increased in pMSCF-miRNA-504-transfected cells, and significantly decreased following transfection with the miRNA-504 inhibitor when compared with controls (Fig. 2). These results suggest that miRNA-504 may promote the proliferation of SMCs by regulating the expression of DNA replication-associated genes, PCMA and RFC4, which are involved in mediating the growth of cells.

Inhibition of miRNA-504 induces apoptosis of SMCs. Apoptosis is a form of cell death characterized by cell shrinkage, chromatin margination, membrane blebbing and nuclear condensation. A number of studies have demonstrated that numerous miRNA sequences regulate apoptosis (35-38). Therefore, in order to determine whether miRNA-504 may be involved in the apoptosis of SMCs, the number of apoptotic cells in pMSCV-miRNA-504 and miRNA-504 inhibitor-transfected SMCs was ascertained using an Annexin V-FITC plus PI staining assay. As shown in Fig. 3, miRNA-504 significantly inhibited the apoptosis of SMCs, as demonstrated by the 4 -fold reduction in apoptosis levels when compared with the controls. By contrast, transfection with the miRNA-504 inhibitor was associated with a 4-fold increase in the degree of apoptosis when compared with controls (Fig. 2). The results indicated that miRNA-504 may regulate the apoptosis of SMCs.

In order to investigate the association between miRNA-504 and the expression of caspase-3, caspase- 9 and Bcl-2, the expression levels of these proteins were determined in SMCs transfected with pMSCV-miRNA-504 and miRNA-504 inhibitors. As shown in Fig. 3, pMSCV-miRNA-504-transfected 

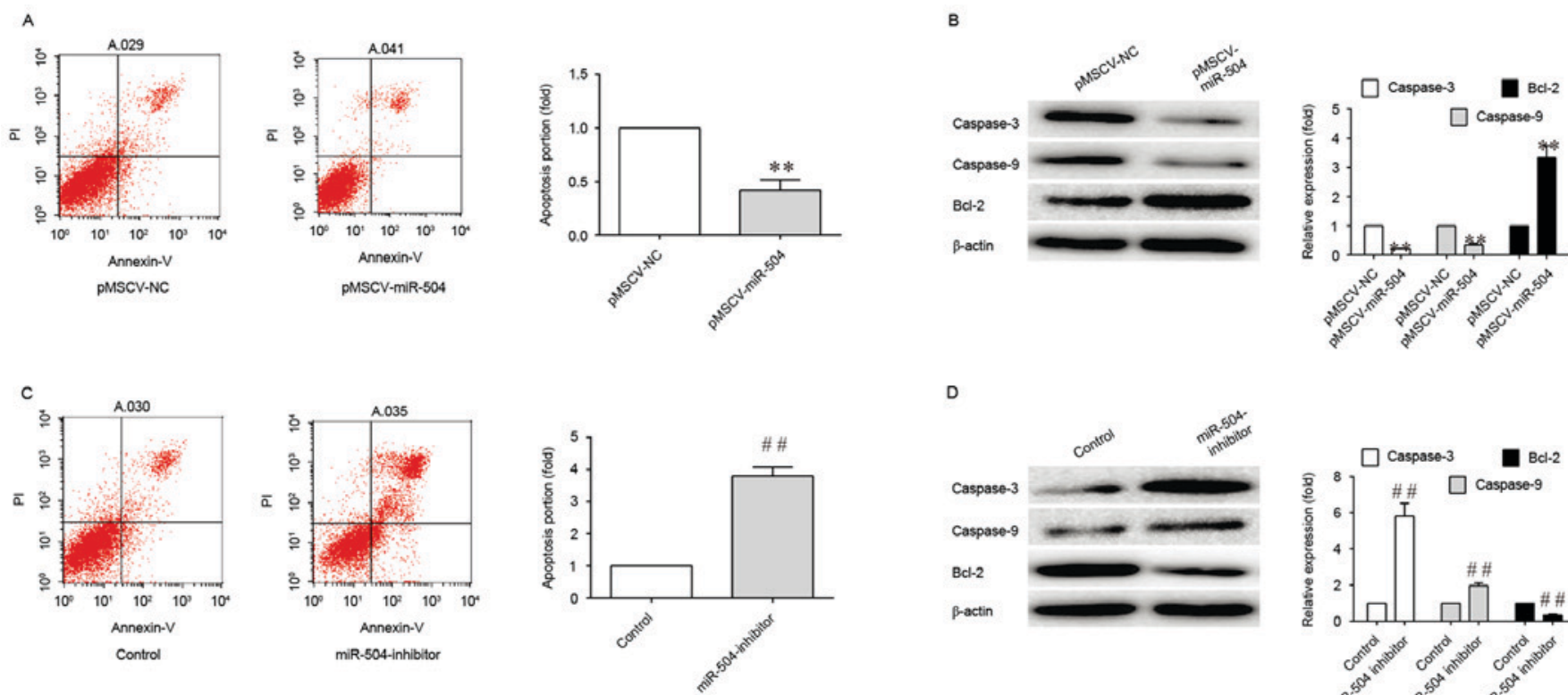

D

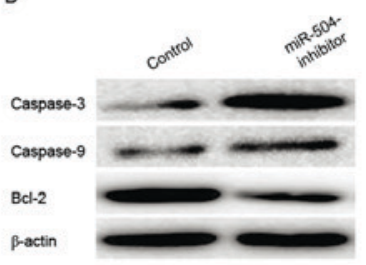

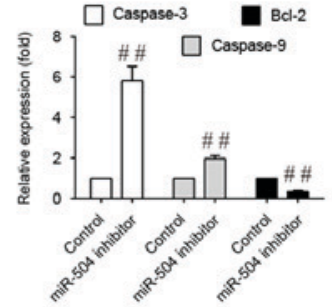

Figure 3. Effect of miRNA-504 expression on the apoptosis of vascular SMCs. (A) Decreased levels of apoptosis were observed in SMCs transfected with pMSCV-miRNA-504. (B) The expression of apoptosis-associated proteins as determined by western blot analysis. (C) Increased levels of apoptosis were observed in SMCs transfected with an miRNA-504 inhibitor. (D) The expression of apoptosis-associated proteins following inhibition of miRNA-504. ${ }^{* *} \mathrm{P}<0.01$ vs. pMSCV-NC; ${ }^{\# \#} \mathrm{P}<0.01$ vs. the control. miRNA, microRNA; SMCs, smooth muscle cells; Bcl-2, B-cell lymphoma-2; PI, propidium iodide.

SMCs exhibited a significant reduction in caspase-3 and caspase-9 protein expression levels, and a significant increase in Bcl-2 expression, when compared with the negative controls. This may have been responsible for the observed increase in apoptosis levels. A significant increase in caspase-3 expression levels was observed in SMCs transfected with the miRNA-504 inhibitor when compared with the controls (Fig. 3). By contrast, the expression levels of the anti-apoptotic gene, Bcl-2 (39), were significantly decreased following transfection with the miRNA-504 inhibitor (Fig. 3). These results indicate that miRNA-504 may serve an anti-apoptotic role in SMCs.

miRNA-504 regulates SMC apoptosis via an p53-dependent mechanism. In order to determine the molecular mechanism underlying the effects of miRNA-504 on SMCs, TargetScan software, which is a bioinformatics tool for miRNA target screening, was employed to search for putative target genes of miRNA-504. p53 was identified as a potential target gene of miRNA-504. In addition, a previous study has reported that overexpression of miRNA-504 inhibits the transcriptional activity of p53 and decreases p53-induced apoptosis and cell-cycle arrest (40). Based on these previous observations, a miRNA-504-based luciferase reporter assay in SMCs was therefore performed in the present study. Comparison of the miRNA-504 and p53 3'-UTR sequences indicated that p53 may be a direct target of miRNA-504 (Fig. 4A). As shown in Fig. 4B and C, the luciferase reporter activity of the wild-type 3'-UTR of p53 was significantly decreased when compared with the luciferase reporter activity of the mutant 3'-UTR sequence following transfection of cells with hsa-miRNA-504 $(\mathrm{P}<0.001$; Fig. 4B). By contrast luciferase reporter expression of wild-type p53 3'-UTR sequences was significantly increased in SMCs following inhibition of miRNA-504.
Western blotting analysis confirmed the decreased and increased expression of p53, as well as its downstream factors, p21 and the pro-apoptotic Bax gene, following transfection of SMCs with the pMSCV-miRNA-504 and miRNA-504 inhibitors, respectively (Fig. 4D). Inhibition of miRNA-504 led to a 3-fold increase in the expression levels of p53, p21 and Bax when compared with the controls ( $\mathrm{P}<0.05$; Fig. 4D). These results suggest that miRNA-504 may regulate SMC growth and apoptosis by directly targeting the 3'-UTR of p53, thereby altering its expression and that of downstream genes involved in cell proliferation and apoptosis.

\section{Discussion}

In normal aortic SMC cells, miRNA-504 is upregulated. In the present study, however, results indicated that miRNA-504 was downregulated in AAA samples of patient, and this suggested that miRNA-504 may be correlated with heritable cardiovascular risk. miRNA-504 may achieve this by regulating the proliferation of SMCs; a critical determinant of vessel function. The ability of SMCs to modulate their phenotype from a quiescent, contractile state to a proliferative, synthetic profile has been implicated in a number of conditions, including atherosclerosis, post-angioplasty restenosis and aortic aneurysm formation (41-45). In view of this important feature of SMCs that is associated with AAA, an in-depth study was performed to investigate the mechanisms underlying the effects of miRNA-504 on SMCs. According to previous studies demonstrating that phenotypic alterations in SMCs are regulated by miRNAs (46-48), miRNA microarray analysis of human SMCs was performed to identify the miRNA sequences involved in AAA. By employing an agnostic whole-genome approach, 12 upregulated and 9 downregulated miRNAs 


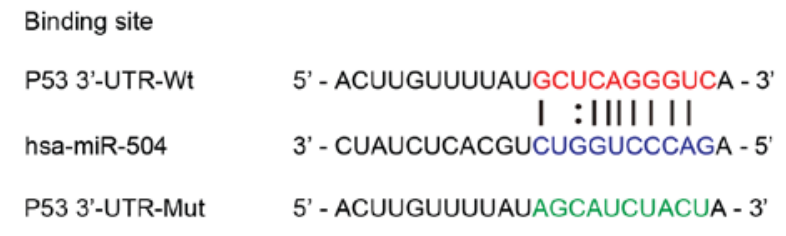

C

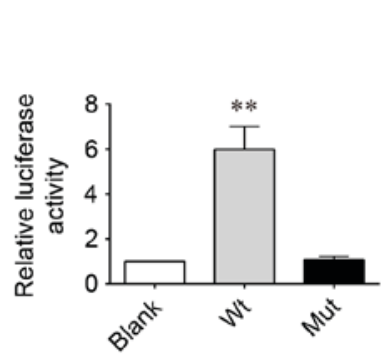

D

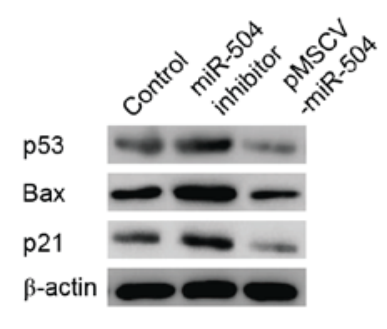

B

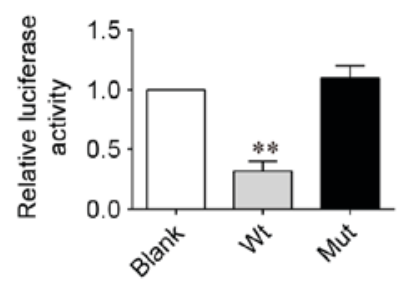

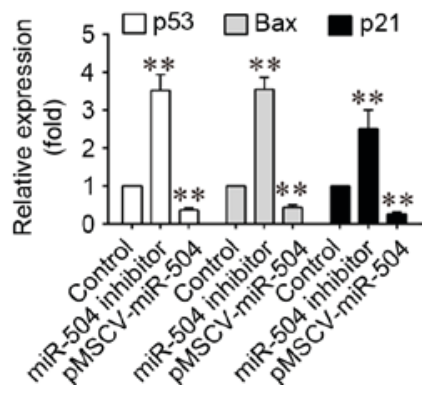

Figure 4. Validation of the direct interation between miRNA-504 and p53. (A) Bioinformatic analysis of the predicted interaction between miRNA-504 and p53. (B) The levels of luciferase activity following transfection of aortic SMCs with p53 3'-UTR-Mut and p53 3'-UTR-Wt sequences together with hsa-miRNA-504 (" P<0.01 vs. blank). (C) The levels of luciferase activity following transfection of aortic SMCs with p53 3'-UTR-Mut and p53 3'-UTR-Wt sequences together with miRNA-504 inhibitor ( ${ }^{* *} \mathrm{P}<0.01$ vs. blank). (D) The protein expression levels of of $\mathrm{p} 53$, $\mathrm{p} 21$ and Bax, as determined by western blotting $($ (** $\mathrm{P}<0.01$ vs. control). miRNA, microRNA; SMCs, smooth muscle cells; Wt, wild-type; Mut, mutation; Bax, Bcl-2-like protein 4.

were identified to be significantly differentially expressed in AAA samples. Among these miRNAs, 6 miRNAs exhibited 4- and 6-fold differences in expression in AAA samples when compared with controls. Verification of the expression of these miRNAs using RT-qPCR indicated that miRNA-504 was closely associated with AAA. Subsequent functional investigation of miRNA-504 demonstrated that this miRNA sequence promoted the proliferation of SMCs.

Over the last several years, a number of studies have provided extensive evidence that miRNAs serve important roles in controlling numerous fundamental cellular processes, including aneurysm formation (49). A number of studies have detected frequent alterations in the expression of miRNAs in a variety of human tumors, suggesting that miRNAs may serve a role as a novel class of tumor promoters or suppressors $(50,51)$. Consistent with these observations, the results of the present study demonstrated that miRNA-504 is an essential miRNA that regulates the proliferation of SMCs in AAA. The function of miRNA-504 was examined by transfecting pMSCV-miRNA-504 into SMCs, which led to an increase in SMC proliferation, as well as an increase in the expression levels of the mRNA/DNA replication genes, PCNA and RFC4. Subsequent analysis revealed that miRNA-504 may have promoted the proliferation of SMCs by reducing the expression of caspase 3 and caspase 9. When activated, these enzymes serve a key role in mediating apoptotic signaling events (52). In addition, miRNA-504 augmented the expression levels of the anti-apoptotic gene, Bcl-2.

A number of different physiological death signals, as well as pathological cellular insults, activate the genetically programmed pathway of apoptosis (53). Apoptosis manifests as two major execution programs downstream of the death signal, one of which is the caspase and organelle dysfunction-induced pathway. As part of this pathway, the mitochondrial dysfunction-induced pathway is the best characterized (54). During mitochondrial dysfunction, Bcl-2 resides upstream of irreversible cellular damage and serves a pivotal role in determining cell fate following induction of the mitochondrial dysfunction pathway. The results of the present study suggest that miRNA-504 inhibited the pathway of apoptosis induction, and enhanced the proliferation of SMCs.

It is well known that the basic function of miRNAs is to regulate target genes by facilitating the direct cleavage of target mRNA sequences and/or by suppressing protein synthesis, which depends on the degree of complementarity of miRNAs with the 3'-UTR of target gene mRNA (55). In present study, p53 was selected as the target gene for miRNA-504 according to TargetScan software. Luciferase reporter assays confirmed that miRNA-504 targets the 3'-UTR of p53, and that p53 expression was significantly downregulated by miRNA-504 overexpression. A number of previous studies have revealed that the function of p53 as an anti-oncogene is short-lived, as it is maintained at low levels by rapid degradation through the mouse double minute 2 homolog, which is a p53-specific E3 ubiquitin ligase $(56,57)$. Activated p53 induces the transcription of target genes, such as p21, to initiate various cellular responses, including cell cycle arrest, apoptosis or senescence. The results of the current study indicated that overexpression of miRNA-504 inhibited the transcriptional activity of p53 and potentially decreased p53-induced apoptosis.

In conclusion, the present study investigated the function of miRNA-504 in SMCs from patients with AAA, and demonstrated that miRNA-504 increased the growth of aortic SMCs and inhibited apoptosis. Therefore, miRNA-504 may 
function as an anti-neoplastic factor in AAA. Investigating the role of miRNA-504 in SMCs from patients with AAA further, may provide an improved understanding of the molecular mechanisms underlying AAA development. These results would provide important information and a wider perspective on AAA intervention, prevention and treatment strategies.

\section{Acknowledgements}

The current study was financially supported by the Natural Science Foundation of Heilongjiang Province (grant no. H201387) and Heilongjiang Postdoctoral Fund (LBH-Z16159).

\section{References}

1. Sakalihasan N, Limet R and Defawe OD: Abdominal aortic aneurysm. Lancet 365: 1577-1589, 2005.

2. Boddy AM, Lenk GM, Lillvis JH, Nischan J, Kyo Y and Kuivaniemi H: Basic research studies to understand aneurysm disease. Drug News Perspect 21: 142-148, 2008.

3. Curci JA and Thompson RW: Adaptive cellular immunity in aortic aneurysms: Cause, consequence, or context? J Clin Invest 114: 168-171, 2004.

4. Svensjo S, Björck M and Wanhainen A: Update on screening for abdominal aortic aneurysm: A topical review. Eur J Vasc Endovasc Surg 48: 659-667, 2014.

5. Kuivaniemi $\mathrm{H}$ and Elmore JR: Opportunities in abdominal aortic aneurysm research: Epidemiology, genetics, and pathophysiology. Ann Vasc Surg 26: 862-870, 2012.

6. Shimizu K, Mitchell RN and Libby P: Inflammation and cellular immune responses in abdominal aortic aneurysms. Arterioscler Thromb Vasc Biol 26: 987-994, 2006.

7. Daugherty A and Cassis LA: Mouse models of abdominal aortic aneurysms. Arterioscler Thromb Vasc Biol 24: 429-434, 2004.

8. Rowe VL, Stevens SL, Reddick TT, Freeman MB, Donnell R, Carroll RC and Goldman MH: Vascular smooth muscle cell apoptosis in aneurysmal, occlusive, and normal human aortas. J Vasc Surg 31: 567-576, 2000.

9. Allaire E, Muscatelli-Groux B, Mandet C, Guinault AM, Bruneval P, Desgranges $\mathrm{P}$, Clowes $\mathrm{A}$, Méllière $\mathrm{D}$ and Becquemin JP: Paracrine effect of vascular smooth muscle cells in the prevention of aortic aneurysm formation. J Vasc Surg 36 1018-1026, 2002.

10. Choke E, Cockerill G, Wilson WR, Sayed S, Dawson J, Loftus I and Thompson MM: A review of biological factors implicated in abdominal aortic aneurysm rupture. Eur J Vasc Endovasc Surg 30: 227-244, 2005.

11. Rotmans JI, Velema E, Verhagen HJ, Blankensteijn JD, de Kleijn DP, Stroes ES and Pasterkamp G: Matrix metalloproteinase inhibition reduces intimal hyperplasia in a porcine arteriovenous-graft model. J Vasc Surg 39: 432-439, 2004.

12. Kuivaniemi H, Platsoucas CD and Tilson MD III: Aortic aneurysms: An immune disease with a strong genetic component. Circulation 117: 242-252, 2008.

13. Abdul-Hussien H, Hanemaaijer R, Kleemann R, Verhaaren BF, van Bockel JH and Lindeman JH: The pathophysiology of abdominal aortic aneurysm growth: Corresponding and discordant inflammatory and proteolytic processes in abdominal aortic and popliteal artery aneurysms. J Vasc Surg 51: 1479-1487, 2010.

14. Lenk GM, Tromp G, Weinsheimer S, Gatalica Z, Berguer R and Kuivaniemi H: Whole genome expression profiling reveals a significant role for immune function in human abdominal aortic aneurysms. BMC Genomics 8: 237, 2007.

15. Pahl MC, Derr K, Gäbel G, Hinterseher I, Elmore JR, Schworer CM, Peeler TC, Franklin DP, Gray JL, Carey DJ, et al: MicroRNA expression signature in human abdominal aortic aneurysms. BMC Med Genomics 5: 25, 2012.

16. Stather P, Sylvius N, Sidloff D, Dattani N, Verissimo A, Wild JB, Butt HZ, Choke E, Sayers RD and Bown MJ: Identification of microRNAs associated with abdominal aortic aneurysms and peripheral arterial disease. Br J Surg 102: 755-766, 2015.
17. Maegdefessel L, Spin JM, Raaz U, Eken SM, Toh R, Azuma J, Adam M, Nakagami F, Heymann HM, Chernogubova E, et al: miR-24 limits aortic vascular inflammation and murine abdominal aneurysm development. Nature Commun 5: 5214, 2014.

18. Angaji SA, Hedayati SS, Poor RH, Madani S, Poor SS and Panahi S: Application of RNA interference in treating human diseases. J Genet 89: 527-537, 2010.

19. Bartel DP: MicroRNAs: Genomics, biogenesis, mechanism, and function. Cell 116: 281-297, 2004.

20. Ambros V: The functions of animal microR NAs. Nature 431: 350-355, 2004.

21. Kong W, Zhao JJ, He L and Cheng JQ: Strategies for profiling microRNA expression. J Cell Physiol 218: 22-25, 2009.

22. Gartel AL and Kandel ES: miRNAs: Little known mediators of oncogenesis. Semin Cancer Biol 18: 103-110, 2008.

23. Calin GA, Sevignani C, Dumitru CD, Hyslop T, Noch E, Yendamuri S, Shimizu M, Rattan S, Bullrich F, Negrini M and Croce CM: Human microRNA genes are frequently located at fragile sites and genomic regions involved in cancers. Proc Natl Acad Sci USA 101: 2999-3004, 2004.

24. Garzon R, Fabbri M, Cimmino A, Calin GA and Croce CM: MicroRNA expression and function in cancer. Trends Mol Med 12: 580-587, 2006.

25. Kong YW, Ferland-McCollough D, Jackson TJ and Bushell M: microRNAs in cancer management. Lancet Oncol 13: e249-e258, 2012.

26. Lee KH, Chen YL, Yeh SD, Hsiao M, Lin JT, Goan YG and Lu PJ: MicroRNA-330 acts as tumor suppressor and induces apoptosis of prostate cancer cells through E2F1-mediated suppression of Akt phosphorylation. Oncogene 28: 3360-3370, 2009.

27. Friedman RC, Farh KK, Burge CB and Bartel DP: Most mammalian mRNAs are conserved targets of microRNAs. Genome Res 19: 92-105, 2009.

28. Krek A, Grün D, Poy MN, Wolf R, Rosenberg L, Epstein EJ, MacMenamin P, da Piedade I, Gunsalus KC, Stoffel M and Rajewsky N: Combinatorial microRNA target predictions. Nat Genet 37: 495-500, 2005.

29. Agarwal V, Bell GW, Nam JW and Bartel DP: Predicting effective microRNA target sites in mammalian mRNAs. Elife 4, 2015.

30. Livak KJ and Schmittgen TD: Analysis of relative gene expression data using real-time quantitative PCR and the 2(-Delta Delta C(T)) method. methods 25: 402-408, 2001.

31. Su Z, Yang Z, Xu Y, Chen Y and Yu Q: MicroRNAs in apoptosis, autophagy and necroptosis. Oncotarget 6: 8474-8490, 2015.

32. Wang Q, Morgan S, Ren J and Liu B: Abstract 295: Macrophages induce apoptosis of arterial smooth muscle cells via a monocyte chemoattractant protein-1 (MCP-1) mediated cytotoxicity. Arteriosc Thromb Vasc Biol 33: A295, 2013.

33. Dietrich DR: Toxicological and pathological applications of proliferating cell nuclear antigen (PCNA), a novel endogenous marker for cell proliferation. Crit Rev Toxicol 23: 77-109, 1993.

34. Reynolds N, Fantes PA and MacNeill SA: A key role for replication factor $\mathrm{C}$ in DNA replication checkpoint function in fission yeast. Nucleic Acids Res 27: 462-469, 1999.

35. Maalouf SW, Liu WS and Pate JL: MicroRNA in ovarian function. Cell Tissue Res 363: 7-18, 2016.

36. Singh DK, Bose S and Kumar S: Role of microRNA in regulating cell signaling pathways, cell cycle, and apoptosis in non-small cell lung cancer. Curr Mol Med: Apr 29, 2016 (Epub ahead of print).

37. Sherrard R, Luehr S, Holzkamp H, McJunkin K, Memar N and Conradt $\mathrm{B}$ : miRNAs cooperate in apoptosis regulation during $C$. elegans development. Genes Dev 31: 209-222, 2017.

38. Zhang Y, Liu W, Wang H, Xu B, Li H and Cao X: miRNA-126b regulated apoptosis of the human tongue carcinoma cell line Tca8113-P60 via P38 signaling pathway. Int J Clin Exp Med 10: 2654-2659, 2017

39. Gross A, McDonnell JM and Korsmeyer SJ: BCL-2 family members and the mitochondria in apoptosis. Genes Dev 13: 1899-1911, 1999.

40. Soutto M, Chen Z, Saleh MA, Katsha A, Zhu S, Zaika A, Belkhiri A and El-Rifai W: TFF1 activates p53 through down-regulation of miR-504 in gastric cancer. Oncotarget 5: 5663-5673, 2014

41. Thompson RW, Liao S and Curci JA: Vascular smooth muscle cell apoptosis in abdominal aortic aneurysms. Coron Artery Dis 8: 623-631, 1997.

42. Inoue $\mathrm{T}$ and Node $\mathrm{K}$ : Molecular basis of restenosis and novel issues of drug-eluting stents. Circ J 73: 615-621, 2009.

43. Doran AC, Meller N and McNamara CA: Role of smooth muscle cells in the initiation and early progression of atherosclerosis. Arterioscler Thromb Vasc Biol 28: 812-819, 2008. 
44. Cai X: Regulation of smooth muscle cells in development and vascular disease: Current therapeutic strategies. Expert Rev Cardiovasc Ther 4: 789-800, 2006.

45. Hoshina K, Koyama H, Miyata T, Shigematsu H, Takato T, Dalman RL and Nagawa H: Aortic wall cell proliferation via basic fibroblast growth factor gene transfer limits progression of experimental abdominal aortic aneurysm. J Vasc Surg 40: $512-518,2004$

46. Scirocco A, Matarrese P, Carabotti M, Ascione B, Malorni W and Severi C: Cellular and molecular mechanisms of phenotypic switch in gastrointestinal smooth muscle. J Cell Physiol 231: 295-302, 2016

47. Charolidi $\mathrm{N}$ and Carroll VA: Hypoxia and pulmonary hypertension. In: Hypoxia and Human Diseases. InTech, 2017.

48. Pei H, Tian C, Sun X, Qian X, Liu P, Liu W and Chang Q: Overexpression of microRNA-145 promotes ascending aortic aneurysm media remodeling through TGF- $\beta 1$. Eur J Vasc Endovasc Surg 49: 52-59, 2015.

49. Kin K, Miyagawa S, Fukushima S, Shirakawa Y, Torikai K, Shimamura K, Daimon T, Kawahara Y, Kuratani T and Sawa Y: Tissue-and plasma-specific microRNA signatures for atherosclerotic abdominal aortic aneurysm. J Am Heart Assoc 1: e000745, 2012
50. Cohn DE, Fabbri M, Valeri N, Alder H, Ivanov I, Liu CG, Croce CM and Resnick KE: Comprehensive miRNA profiling of surgically staged endometrial cancer. Am J Obstet Gynecol 202: 656.e1-e8, 2010.

51. Kwak PB, Iwasaki S and Tomari Y: The microRNA pathway and cancer. Cancer Sci 101: 2309-2315, 2010.

52. Stadelmann C and Lassmann H: Detection of apoptosis in tissue sections. Cell Tissue Res 301: 19-31, 2000.

53. Vaux DL and Korsmeyer SJ: Cell death in development. Cell 96: 245-254, 1999.

54. Green DR and Reed JC: Mitochondria and apoptosis. Science 281: 1309-1312, 1998.

55. Chekulaeva M and Filipowicz W: Mechanisms of miRNAmediated post-transcriptional regulation in animal cells. Curr Opin Cell Biol 21: 452-460, 2009.

56. Harris SL and Levine AJ: The p53 pathway: Positive and negative feedback loops. Oncogene 24: 2899-2908, 2005.

57. Itahana K, Mao H, Jin A, Itahana Y, Clegg HV, Lindström MS, Bhat KP, Godfrey VL, Evan GI and Zhang Y: Targeted inactivation of Mdm2 RING finger E3 ubiquitin ligase activity in the mouse reveals mechanistic insights into p53 regulation. Cancer Cell 12: 355-366, 2007. 